\title{
Detection and Classification of Explosive Compounds in Presence of Interferents Utilizing Laser Ion Mobility Spectrometry
}

\author{
A. Langmeier, C. Oberhüttinger, W. Heep, H. Oberpriller, S. Beer, M. Kessler, J. Göbel, G. Müller \\ EADS Innovation Works Germany \\ 81663 München
}

\begin{abstract}
Ion Mobility Spectrometers (IMS) are frequently used to screen airline passengers and their luggage for explosives and explosive by-products. Traditionally, in IMS technology different kinds of molecules are distinguished on account of their different ion mobilities in a weak electric field. In this paper we report on methods of resolution enhancement applicable to IMS drift time spectra. We find that a robust classification of trace explosives can be obtained by algorithmic resolution enhancement of the raw drift time spectra and by an additional parameterisation of the desorption function of the sampled explosives.
\end{abstract}

\section{Introduction to IMS}

Ion Mobility Spectrometry (IMS) is a trace-detection technology which is based on mobility measurement of charged particles and ions under atmospheric pressure conditions [1]. The charge is applied to the particles either by radioactive radiation, electron attachment, field-, photo- or electrospray ionisation. After ionisation, the particles or molecules undergo an acceleration process under the influence of a linear electrical drift field towards an ion collector electrode. Different kinds of molecules can be distinguished on account of their characteristic arrival times at the collector electrode, which depends on the molecular size and/or cross-sections. Important applications of IMS are in the fields of analytical chemistry [2] or explosives detection [3].

\section{LIMS Hardware Instrumentation}

The work presented here was performed with a laser ion mobility spectrometer (LIMS) prototype developed at EADS-IW [4]. Here, in contrast to classical IMS systems, a laser, instead of a radioactive ${ }^{63} \mathrm{Ni}$ source is used for the ionisation of analyte, dopant and calibrant substances as this is a very selective and soft ionisation method [5]. The laser is a fixed wavelength UV laser based on a flash-lamp pumped Nd:YAG crystal with a wavelength of $266 \mathrm{~nm}$. The studies were performed with a laser power of $20 \mathrm{~mW}$ and a pulse duration time of $5 \mathrm{~ns}$. A schematic view of the instrument is shown in Fig. 1.
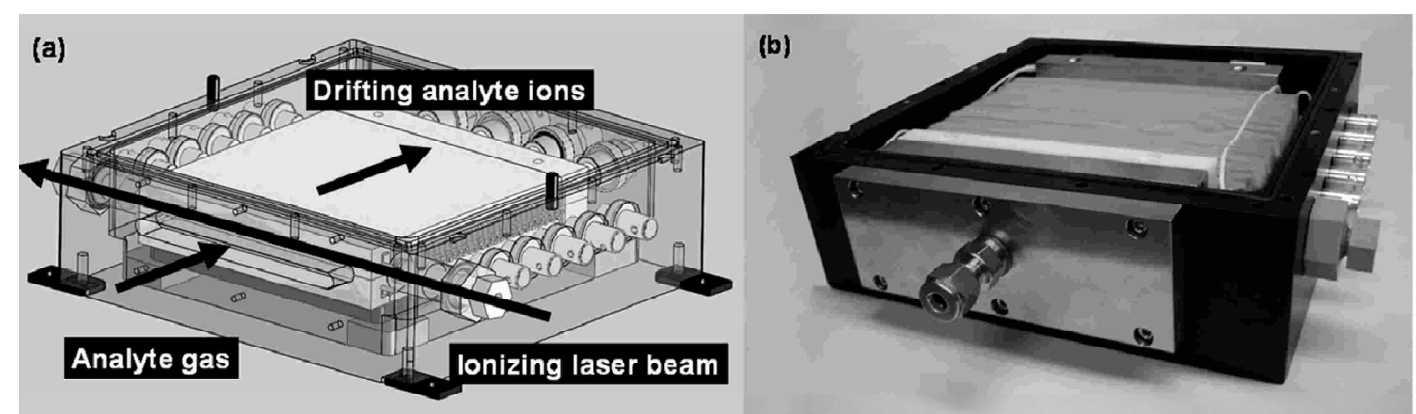

Fig.1: Schematic design of the LIMS drift tube; (b) stacked-design drift tube assembled within the LIMS drift tube package [6].

The drift tube has a drift length of $10.6 \mathrm{~cm}$ consisting of ceramic drift plates with integrated electrode patterns. An electrical field of $E=248 \mathrm{~V} / \mathrm{cm}$ was applied for the measurements. We have used a rectangular drift tube design as it allows for a relatively long interaction path of the UV laser beam with the analyte molecules in the entrance region of the LIMS drift tube. Pressurized synthetic air with a flow rate of $100 \mathrm{sccm}$ was used to generate the carrier and drift gas flows. Throughout the experiments, the inlet and drift tube temperatures were set at $150^{\circ} \mathrm{C}$ and $40^{\circ} \mathrm{C}$, respectively. The desorber heater temperature was kept constant and varied between $80^{\circ} \mathrm{C}$ and $280^{\circ} \mathrm{C}$, depending on the type of explosive. 
Explosive standard solutions were procured from Restec company. The explosive compounds were solved in acetonitrile or methanol with a concentration of $1000 \mu \mathrm{g} / 1000 \mu \mathrm{l}$. For measurement, the explosive/interferent solutions were applied to sampling swabs. A syringe device was used for dosing the explosives in the 1 to $500 \mathrm{ng}$ range. After preparation, the swabs were heated at a constant temperature for $60 \mathrm{~s}$ to allow the evaporation of the explosives and the beginning depletion of the swabs to be followed in sufficient detail.

\section{Results}

Spectral analysis of several explosive compounds was performed. The explosives investigated included substances with aromatic (2,4-DNT, 2,6-DNT, 2,4,6-TNT, DNB, TNB) and non-aromatic ring structures (Tetryl, HMX) as well as others with aliphatic backbone structures (PETN, GTN and TATP). All of them were detectable with LIMS with detection limits ranging from $0.5 \mathrm{ng}$ (TNT) to approximately $20 \mathrm{ng}$ (GTN).

In total, we investigated 21 interferent compounds for their LIMS response. These substances belonged to five different chemical groups which are all known to interfere with the detection of TNT, RDX \& HMX, PETN \& EGDN using conventional radioactive IMS [7]. The five groups are (1) acidic fog (nitrated phenols) from air pollution, (2) tobacco by-products, (3) synthetic fragrances used in perfumes and scents, (4) insecticides and fertilizers and (5) vasodilators in drugs for heart diseases. The 21 studied compounds were chosen because of their similarity to explosives with regard to hydrocarbon backbone structure and functional side groups. The suspected contaminants have at least one nitro functional group, with the exception of methyl parathion. The nitro functional group is the primary electronwithdrawing group present in most explosive compounds. Six of these compounds could not be detected with LIMS. Those that could be detected, exhibited detection limits in the $\mu \mathrm{g}$ range and thus were detectable with sensitivities at least ten times lower than those of the corresponding explosives. Additionally, these detectable interferents caused no problems regarding PLS classification as they differed in their spectral characteristics from the corresponding explosives.

\section{Algorithmic System and Spectral Features}

All spectra presented here were acquired in negative ion mode. The data acquisition frequency was 50 $\mathrm{kHz}$. The scan period was set at $100 \mathrm{~ms}$. Most of the results presented here were achieved by offline analysis with a Matlab based spectral analysis system.

Currently our resolution in raw spectra is around $R \sim 30$. The inherently low spectral resolution in ion mobility spectrometers - at least as compared to mass spectrometers - requires additional measures to classify explosives and to distinguish them from their corresponding interferents. This can be done using phenomenological methods or by extracting specific spectral features from the drift time spectra. In the first method, the entire spectra are used for classification, whereas in the second specific spectral features such as peak positions, peak shape etc. are compared with features from a database. The main substance-specific features in the drift time spectrum - the molecule-specific drift time and the corresponding $\mathrm{K}_{0}$ value - can be estimated up to a certain resolution which is often insufficient for classification. In order to ameliorate this situation we used a deconvolution algorithm proposed by Lucy [8]. With this deconvolution, our base instrumental resolution of $R \sim 30$ could be improved to $R \sim 50$.

Fig. 2 shows a drift time spectrum of 2,4-Dinitrophenol and 2,4,6-TNT before and after deconvolution. The improvement in spectral resolution is immediately visible.

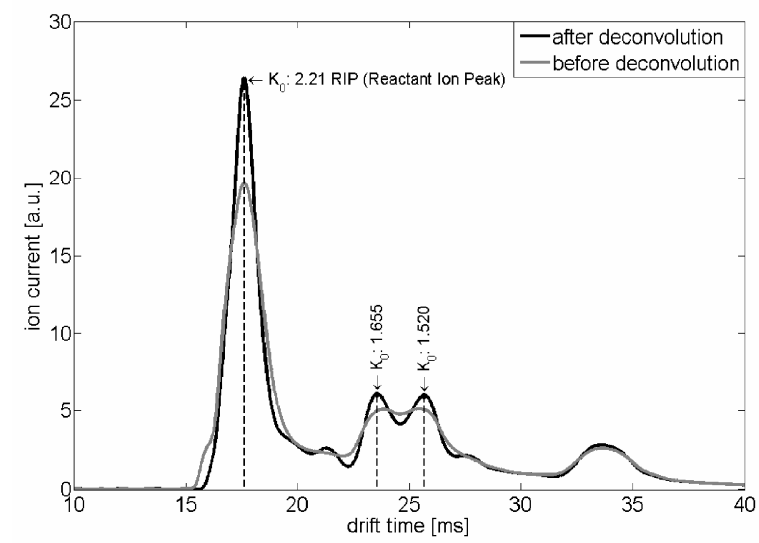

Fig.2: The effect of deconvolution to the drift time spectrum of 2,4-DNP and 2,4,6-TNT. The broad feature at about $33 \mathrm{~ms}$ corresponds to the unresolved dimers of both compounds. 
Deconvolution also improves the statistical (phenomenological) classification with a Partial Least Square (PLS) algorithm [9]. Fig. 3 shows a series of PLS classification trained spectra of TNT and those of the traceable nitrated phenols from our interferent list.
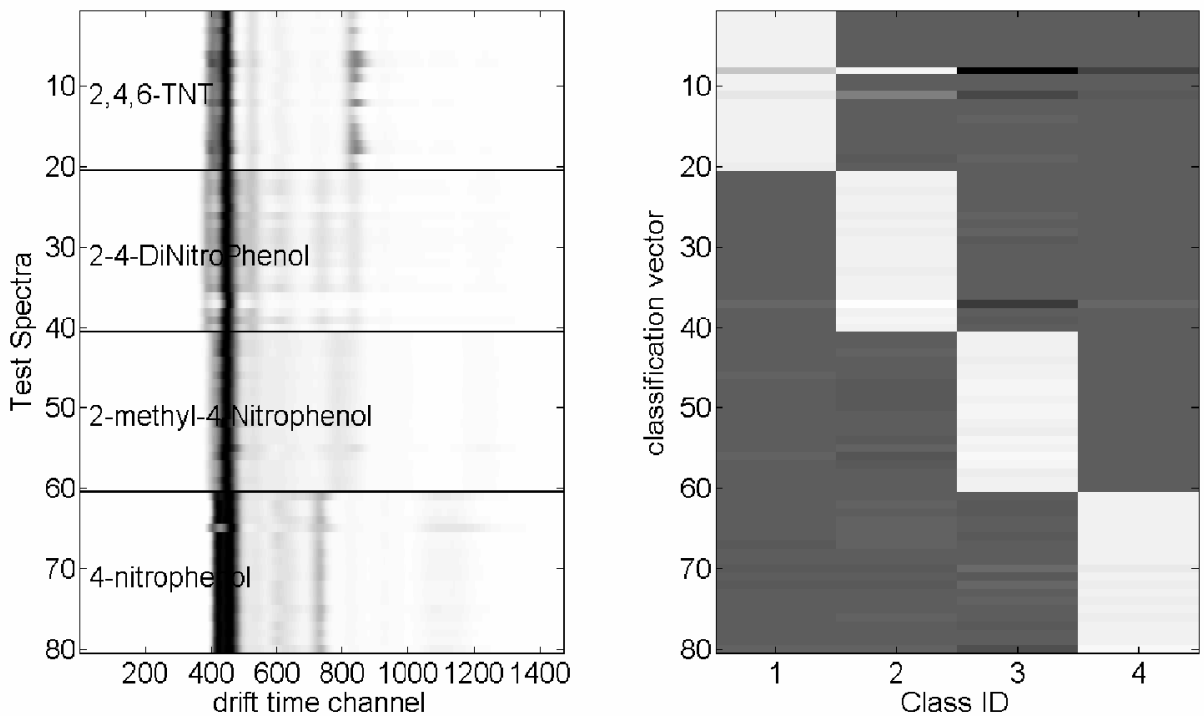

Fig.3: Demonstration of PLS classification with TNT against acidic fog components.

The left template shows randomly choosen test spectra that are background reduced, calibrated and deconvoluted. These give input to the PLS classification which was trained with a disjunct training data set. The right template shows the corresponding classification vector. The 20 TNT test spectra are correctly classified except for two of them.

\section{Desorption Features}

Even after an algorithmic resolution enhancement, the measurement of the peak positions is often insufficient to identify and distinguish different analytes. For example, TNT and TNB have a very similar chemical structure and almost identical $\mathrm{K}_{0}$ values $\left(\mathrm{K}_{0, \mathrm{TNT}}=1.54, \mathrm{~K}_{0, \mathrm{TNB}}=1.53\right)$ and thus are not resolvable with our present spectral resolution. Desorption features may be used to distinguish substances with nonresovable drift time values, which is demonstrated in Fig. 4.

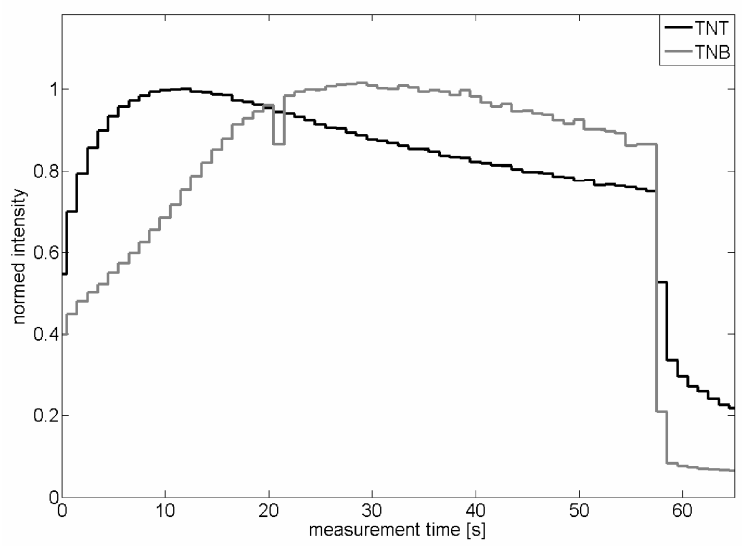

Fig. 4: The normalised desorption histories of TNT and TNB. TNT and TNB can be distinguished on the basis of their desorption curves. Start and end of desorption, $60 \mathrm{~s}$ later, can be clearly seen.

(a) Derivatives

There are several extractable parameters of the desorption functions available for classification. One of the seemingly obvious approaches, namely the use of first derivatives to gain distingushing features, fails within the desired fast classification time of 10 seconds. This is due to the fact that during the first seconds of desorption, the rise in intensity shows a collective behaviour in all spectral components, caused by a change in the air flow and the availability of dopant and consecutive reactant ions. The 
numerical estimation of curvature values and the necessary calculation of second derivatives appeared to be too noisy.

(b) Mathematical modelling

The direct parametrisation with a modeling function yields good results, but is computationally expensive and requires a non-linear optimisation algorithm that is demanding on real time applications due to the unknown number of iterations needed for convergence. This type of algorithm imperatively requires a set of start parameters that lie near the solution to guarantee convergence. We found that a fit with a asymmetric Gauss function with four parameters gives satisfactory results, as shown in Fig. 5. Desorption curves can be characterized with 4 parameters (features). We also tested fits with a log-normal function with inferior outcomes. Parameterisation of this kind needs also extended measurements (about $50 \mathrm{~s}$ ) and is therefore not suitable for short measurement cycles.

$$
\begin{aligned}
& G_{1}=1 . /\left(1+\exp \left(-(x-\mu) / \sigma_{1}\right)\right) \\
& G_{2}=1 . /\left(1+\exp \left(-(x-\mu) / \sigma_{2}\right)\right) \\
& G(x, \sigma, \mu, f)=f \cdot\left(G_{1} \cdot\left(1-G_{2}\right)\right)
\end{aligned}
$$

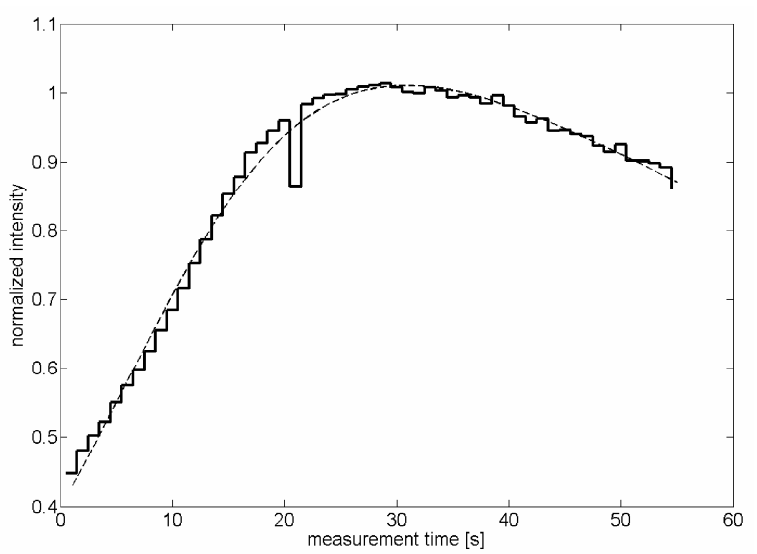

Fig.5: Fit of a asymmetric Gauss function to the desorption curve of TNB.

(c) Rise times and extrapolated desorption times

To avoid the computational and alghorithmically demanding non-linear optimisation, a third method was tested. We estimated the total desorption time by linearly extrapolating the decaying slope of the logarithm of the desorption function. Unfortunately, this method also requires relatively long measurements of around $60 \mathrm{~s}$ to gain resonable results for our set of explosive compounds. It may be more effective for fast desorbing substances. The estimated desorption time is defined as the time at which the intensity is equal to 0.05 , e.g. 5 percent of the peak intensity. Fig. 6 shows the normalized desorption history of TNT and the linear extrapolation The fall times are a promising feature to distinguish substances with identical drift times.

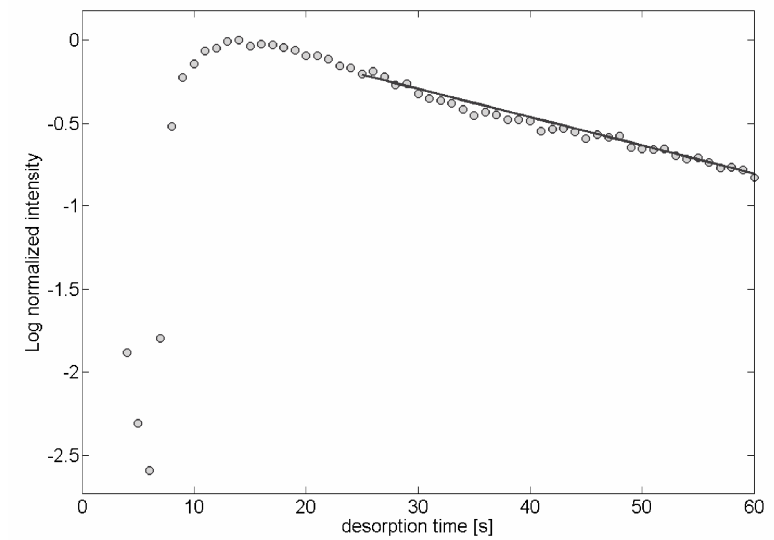

Fig.6: Linear extension of the log of the desorption curve of TNT. 


\section{Conclusions}

Spectral analysis of several major explosive compounds with aromatic (2,4-DNT, 2,6-DNT, 2,4,6-TNT, DNB, TNB), non-aromatic ring structures (Tetryl, HMX) and aliphatic hydrocarbon backbone structures (PETN, GTN and TATP) was performed. All of them could be detected with LIMS with detection limits ranging from $0.5 \mathrm{ng}$ for TNT up to about $20 \mathrm{ng}$ for GTN.

The sensitivity of the LIMS traceable interferents was at least ten times lower than for explosives, with detection limits in the $\mu \mathrm{g}$ range. Six out of 21 interferent compounds could not be detected at all, and therefore did not pose any problem for explosive detection. Additionally, the remaining interferents caused no problems regarding PLS classification as their spectral characteristics are distinguishable from those of the explosives.

Algorithmic enhancement of the LIMS base instrumental resolution has been achieved. Algorithmic enhancement improved the resolution of drift time peaks which were not separated in the raw spectra. Deconvolution also improved the resolution in statistical classification (PLS).

Analysis of desorption curves allows for the extraction of additional spectral features. These can be used to distinguish substances with similar drift times, e.g. TNB and TNT. The most promising way of gaining such features was the estimation of the rise and decay times from the observed desorption curves.

\section{Acknowledgements}

The authors gratefully acknowledge continuous interest and support by Dr. Valerie Manning and Sam Wyman. The authors are also indebted to Al Mercado for clarifying discussions about interferent substances.

\section{References:}

[1] Eiceman GA, Karpas Z (2005) Ion Mobility Spectrometry. CRC Press, Boca Raton

[2] Louis RHSt, Hill HH Jr (1990) "Ion Mobility Spectrometry in analytical chemistry", Crit Rev Anal Chem, Vol. 21, pp. 321

[3] Ewing RG, Atkinson DA et al (2001) "A critical review of ion mobility spectrometry for the detection of explosives and explosive related compounds", Talanta, Vol. 54, pp. 515

[4] Goebel J, Kessler M, Langmeier A (2009) "A novel Laser lon Mobility Spectrometer", ISOEN 2009, Brescia, (submitted)

[5] Lubman DM, Kronick MN (1983) "Multiwavelength-selective ionization of organic compounds in an ion mobility spectrometer", Anal Chem, Vol. 55, pp. 867

[6] Oberhuettinger C, Langmeier A et al (2008) "Hydrocarbon Detection Using Laser Ion Mobility Spectrometry", Int J Ion Mobil Spectrom, (submitted)

[7] Matz LM, Tornatore PS et al (2001) "Evaluation of suspected interferents for TNT detection by ion mobility spectrometry", Talanta, Vol. 54, pp. 171

[8] Lucy LB (1974) "An iterative technique for the rectification of observed distributions", Astron J, Vol.79, pp. 745

[9] Haenlein M, Kaplan AM (2004) "A beginner's guide to partial least squares (PLS) analysis", Underst Stat, Vol. 3, pp. 283 